Überlebensraten noch bei $20,8 \%$ bzw. $12,2 \%$. Das mediane Gesamtüberleben betrug 11,2 Monate für die Patienten unter Ipilimumab plus Dacarbazin und 9,1 Monate für die Patienten unter Dacarbazin $(\mathrm{p}<0,0009)$. In allen Subgruppen war das Gesamtüberleben nach der Kombinationstherapie verlängert. Das mediane krankheitsfreie Überleben war mit 2,8 und 2,6 Monaten vergleichbar. Die Zeit für das Ansprechen des Tumors auf die Therapie
(WHO-Kriterien, nach Größe und Anzahl der Läsionen im Vergleich zum Studienbeginn) war im Kombinationsarm signifikant länger (19,3 vs. 8,1 Monate).

Grad-3/4-Nebenwirkungen traten unter Ipilimumab plus Dacarbazin häufiger auf (56 vs. 27\%). Das Nebenwirkungsprofil von Ipilimumab aus früheren Studien habe sich mit dieser Studie bestätigt, so Wolchok. Die häufigsten Nebenwirkungen waren Pruritus (29,6 vs. 8,8\%),
Hautausschlag (24,7 vs. $6,8 \%)$, Diarrhö ( 36,4 vs. $24,7 \%$ ), Colitis ( 4,5 vs. $0,4 \%$ ) und Anstieg der Transaminasen (ALT: 21,9vs. $0,8 \%$ und AST: 18,2 vs. $1,2 \%)$.

Andrea Warpakowski

Wolchok JD. Phase III randomized study of ipilimumab (IPI) plus dacarbazine (DTIC) versus DTIC alone as first-line treatment in patients with unresectable stage III or IV melanoma. 5.6.2011

\title{
ASC(T) Targeted-Therapie bei Mutation
}

Patienten mit einer Mutation im BRAFGen profitieren von der Monotherapie mit Vemurafenib: Nach vorläufigen Daten verlängerte der BRAF-Inhibitor im Vergleich zu Dacarbazin Gesamtüberleben und progressionsfreies Überleben. Bei etwa der Hälfte der Melanom-Patienten weist der Tumor die aktivierende Mutation V600E im BRAF-Gen auf. Dieses Gen spielt eine Schlüsselrolle beim Zellwachstum und bei der Zellproliferation. Vemurafenib hemmt spezifisch die mutierte BRAF-Kinase und stoppt damit das Tumorwachstum.

Die Wirksamkeit des BRAF-Inhibitors wurde in einer randomisierten offenen Phase-III-Studie an 675 bisher nicht behandelten Patienten mit fortgeschrittenem, inoperablen metastasiertem Mela- nom mit der Mutation V600E untersucht. Die Daten der geplanten Zwischenanalyse nach einem medianen Follow-up von drei Monaten präsentierte Dr. Paul Chapman, New York.

Die Patienten erhielten entweder Vemurafenib (960 mg zweimal täglich als Tablette) oder Darcarbazin $\left(1.000 \mathrm{mg} / \mathrm{m}^{2}\right.$ i.v. alle drei Wochen). Gemäß der Zwischenanalyse nach drei Monaten war das Mortalitätsrisiko unter Vemurafenib gegenüber Dacarbazin um $63 \%$ und die Wahrscheinlichkeit einer Tumorprogression um $74 \%$ verringert. Insgesamt $48,4 \%$ der Patienten sprachen auf die Therapie mit dem BRAF-Inhibitor an und 5,5\% auf die Chemotherapie. Aufgrund der Wirksamkeit von Vemurafenib empfahl das Data and Monitoring Safety Board, dass

den Patienten aus dem Dacarbazin-Arm ein Wechsel zur Vemurafenibtherapie erlaubt sein sollte. Zum Zeitpunkt der Analyse wurden noch $66 \%$ der Patienten im Vemurafenib-Arm und 25\% im Dacarbazin-Arm behandelt.

Die am häufigsten aufgetretenen $\mathrm{Ne}$ benwirkungen unter Vemurafenib waren Keratoakanthome und kutane Spindelzellkarzinome (12 und 6\% vs. jeweils > $1 \%)$, Hautausschlag (8 vs. $0 \%$ ), erhöhte Leberwerte (7 vs. $1 \%$ ), Arthralgie (3 vs. < $1 \%$ ) und Photosensitivität (3 vs. 0\%).

Andrea Warpakowski

Chapman PB. Phase III randomized, openlabel, multicenter trial (BRIM3) comparing BRAF inhibitor vemurafenib with dacarbazine (DTIC) in patients with V600EBRAmutated melanoma. 5.6.2011

(28\%) und Schüttelfrost (22\%). Bei den meisten Patienten wurde eine Reduktion der Tumorgröße beobachtet. Fünf Patienten sprachen mit einer kompletten Remission auf die Therapie an. Insgesamt 80\% der Patienten waren zum Zeitpunkt der Zwischenanalyse noch in der Studie. In der anschließenden randomisierten Phase-II-Studie wird die Dosierung zweimal täglich 2 mg GSK212 und zweimal täglich 150 mg GSK436 weiter untersucht.

Andrea Warpakowski gerte in präklinischen Studien das Auftreten von Resistenzen gegen den BRAFInhibitor, war wirksamer als die Einzelsubstanzen und verhinderte die durch die BRAF-Inhibition hervorgerufenen hyperproliferatorischen Hautläsionen.

Vorläufige klinische Daten zur KombiGSK212 und des oralen BRAF-Inhibitors GSK436 belegen, dass auch diese Therapiestrategie bei fortgeschrittenem Melanom wirkt.

Die Kombination der beiden Kinase-
In der von Dr. Jeffrey Infante, Nashville, vorgestellten Phase-I/II-Studie erhielten 45 nicht vorbehandelte Patienten mit der BRAF-Mutation V600 (davon 43 mit fortgeschrittenem Melanom-Stadium IV) im Rahmen einer Dosiseskalation zweimal täglich 1-2 mg GSK212 und zweimal täglich 75-150 mg GSK 436.

Unter der Kombination traten weniger BRAF-Inhibitor-induzierte squamöse Zellkarzinome $(<1 \%)$ und Hautausschläge (12\%) auf als unter einer BRAF-InhibitorMonotherapie. Weitere häufige Nebenwirkungen waren Fieber (37\%), Fatigue
Infante JR. Phase I/II study to assess safety, pharmacokinetics, and efficacy of the oral MEK 1/2 inhibitor GSK1120212 (GSK212) dosed in combination with the oral BRAF inhibitor GSK2118436 (GSK436). 6.6.2011 